\title{
Suppression of Multiple Ionization of Atomic Ions in Intense Ultrafast Laser Pulses
}

\author{
J. B. Greenwood, I. M. G. Johnston, P. McKenna, and I. D. Williams \\ Department of Physics, Queen's University Belfast, Belfast BT7 1NN, United Kingdom \\ T. R. J. Goodworth, J. H. Sanderson, W. A. Bryan, A. A. A. El-Zein, and W. R. Newell \\ Department of Physics \& Astronomy, University College London, Gower Street, \\ London WClE 6BT, United Kingdom \\ A. J. Langley and E. J. Divall \\ Central Laser Facility, Rutherford Appleton Laboratory, Chilton, Oxfordshire, OX11 OQX, United Kingdom \\ (Received 20 December 2001; published 20 May 2002)
}

\begin{abstract}
The interaction of an intense laser field with a beam of atomic ions has been investigated experimentally for the first time. The ionization dynamics of $\mathrm{Ar}^{+}$ions and Ar neutrals in a $60 \mathrm{fs}, 790 \mathrm{~nm}$ laser pulse have been compared and contrasted at intensities up to $10^{16} \mathrm{~W} \mathrm{~cm}^{-2}$. Our results show that nonsequential ionization from an $\mathrm{Ar}^{+}$target is strongly suppressed compared with that from the corresponding neutral target. We have also observed for the first time the strong field ionization of high lying target metastable levels in the $\mathrm{Ar}^{+}$beam.
\end{abstract}

DOI: $10.1103 /$ PhysRevLett.88.233001

PACS numbers: $32.80 . \mathrm{Fb}$

There is considerable current interest in the interaction of high intensity $\left(10^{13}-10^{18} \mathrm{~W} \mathrm{~cm}^{-2}\right)$ radiation with dilute matter, where the external electric field strength becomes comparable to molecular bond and valance electron binding strengths. Studies have been carried out with targets of atoms, molecules, clusters [1], and most recently molecular ions [2,3], in order to elucidate the new physics observable in this highly nonlinear regime. These include the study of high harmonic generation [4], strong field effects in molecular dissociation, and the enhancement of multiple ionization in atoms [5,6]. Work is currently underway to generate attosecond pulses through harmonic generation $[7,8]$, and coherent $\mathrm{x}$-ray generation could be possible for highly charged ions in the presence of superintense fields $[9,10]$.

Both experiment and theory have shown that, in femtosecond laser pulses, multiple ionization of atoms is enhanced by nonsequential processes $[5,6,11,12]$. The physical model which best describes the observed behavior is known as the "atomic antenna" [13] or "recollision" $[14,15]$ model. In this picture the first electron is removed from the atom by tunneling through the barrier created by interaction of the external field with the atomic potential. This electron is then accelerated in the external field and, depending on the initial phase, can return to the singly charged ion with energies up to $3.17 \mathrm{U}_{P}[6]$, where $\mathrm{U}_{P}$ is the classical ponderomotive energy in the field. Multiple ionization is thus via electron interaction with the ionic core. Evidence for this mechanism includes large reductions in nonsequential ionization rates for circularly polarized fields and more recently from the momentum distribution of the recoil ions [16,17], and coincidence measurements between ions and ejected electrons [18]. However, it has proved difficult to predict quantitative yields for the product ions without using large-scale calculations through the $S$-matrix approach [19] or by obtaining a time-dependent solution of the Schrödinger equation [20].

By including the effects of excitation from the recollision followed by tunneling [21] and taking into account the Coulomb focusing on the active electron [22], better agreement with experiment has been obtained. The importance of multiple returns to the core has been demonstrated experimentally by a reduction in nonsequential ionization when few cycle pulses are used [23].

To fully understand these influences and how they are affected by ionization potentials and different electron correlation dynamics, it is important for experimentalists to undertake studies for a range of targets. All experiments to date have concentrated on neutral targets, mostly on the closed shell configuration of the noble gases. By contrast our experiment is capable of studies using a fast beam of atomic positive ions, in addition to neutral atoms, as a target. Our apparatus has recently been used to yield new and unambiguous insights into the fragmentation dynamics of $\mathrm{H}_{2}^{+}$in a strong laser field [2]. A previous attempt to study the interaction of atomic ions in intense laser fields used in fact neutral targets, but assumed that the ions were formed well before the laser pulse reached its maximum [24].

In this paper we report on the first direct studies of strong field ionization of a positive atomic ion. Since the ion source used to produce the ions also generates long lived excited states, we have been able to study ionization from both the ground state and metastable states. Comparison of the results from an $\mathrm{Ar}^{+}$ion beam and an $\mathrm{Ar}$ gas jet shows that triple ionization of $\mathrm{Ar}$ is more probable than double ionization of $\mathrm{Ar}^{+}$in the nonsequential regime.

A full description of the experimental arrangement and procedure will be given in a future communication, so only the essential details are reported here. A beam of $\mathrm{Ar}^{+}$ions 
was extracted from an oscillating electron-type ion source, momentum selected by a bending magnet, and focused through a differentially pumped chamber to a diameter of $1.5 \mathrm{~mm} \mathrm{FWHM}$ at the point of interaction with the laser beam. A Ti:sapphire laser produced $60 \mathrm{fs}$ pulses at $\lambda=790 \mathrm{~nm}$, at a repetition rate of $10 \mathrm{~Hz}$. The laser beam was focused by a lens using $f / 25$ optics into the ultrahigh vacuum chamber, giving a spot size of $1.6 \times$ $10^{-2} \mathrm{~mm}$ FWHM at the point of interaction. The lens could be computer driven a distance of $25 \mathrm{~mm}$ parallel to the laser beam axis ( $z$ axis), with respect to the optimum focusing position. In this way lower laser intensities could be accessed with increased interaction volumes, enhancing the sensitivity of the instrument as the intensity decreased. Subsequent to interacting with the laser pulse, the fast ion beam was deflected in a $45^{\circ}$ parallel plate analyzer, such that ionized products could be detected in an off-axis channel electron multiplier, while the primary $\mathrm{Ar}^{+}$beam was collected in a well-baffled Faraday cup. Neutral Ar spectra were collected using a Wiley-McLaren-type time of flight mass spectrometer employing an identical optical configuration to that used in the ion beam apparatus [25].

Figure 1a shows a typical $10^{3}$ shot averaged time-offlight spectrum obtained from neutral Ar at an intensity close to the expected limit of the nonsequential regime for production of $\mathrm{Ar}^{3+}$. The ions were extracted through a $0.9 \mathrm{~mm}$ slit, thus restricting detection to the center of the focal volume only. Figure $1 \mathrm{~b}$ shows signal strength from ionization of an $\mathrm{Ar}^{+}$ion beam under similar laser conditions, with the parallel plate analyzer configured to transmit (i) $\mathrm{Ar}^{2+}$ and (ii) $\mathrm{Ar}^{3+}$ ions. After correction for the different interaction volumes in each case, the ratio of $\mathrm{Ar}^{3+}$ to $\mathrm{Ar}^{2+}$ production was found to be $0.038 \pm 0.002$ for the neutral target and $0.007 \pm 0.002$ for the ion target. Thus at this intensity $\mathrm{Ar}^{3+}$ production is strongly suppressed when $\mathrm{Ar}^{+}$rather than $\mathrm{Ar}$ forms the initial target.

Figure 2(a) shows the $\mathrm{Ar}^{2+}$ signal obtained from strong field ionization of the primary $\mathrm{Ar}^{+}$beam, at a maximum intensity of $10^{16} \mathrm{~W} \mathrm{~cm}^{-2}$, as the focusing lens is translated with respect to $z=0$. Each point represents the integral under the $\mathrm{Ar}^{2+}$ peak in the corresponding time-of-flight spectrum. The sharp peak observed at $z=0$, i.e., at the maximum focused intensity, is due to single ionization of ground state $3 s^{2} 3 p^{52} \mathrm{P} \mathrm{Ar}^{+}$ions. However, it is well known that $\mathrm{Ar}^{+}$ions produced in a discharge source have a small fraction of ions populated in metastable states, lying between 16 and $19 \mathrm{eV}$ above the ground state [26]. These long lived states are $3 p^{4} 3 d^{4} D_{7 / 2}(16.4 \mathrm{eV})$, $3 p^{4} 3 d^{4} F_{9 / 2} \quad(17.6 \mathrm{eV}), \quad 3 p^{4} 3 d^{4} F_{7 / 2} \quad(17.7 \mathrm{eV})$, and $3 p^{4} 3 d^{2} F_{7 / 2}(18.5 \mathrm{eV})$. Thus the ionization potentials for the metastable components are approximately $9-11 \mathrm{eV}$, compared with $27.6 \mathrm{eV}$ from the ground state. The two peaks in Fig. 2(b), on either side of the zero position, are due to metastable $\mathrm{Ar}^{+}$ions, displaying the expected profile resulting from the interplay of increasing interaction volume with decreasing intensity as the lens moves away
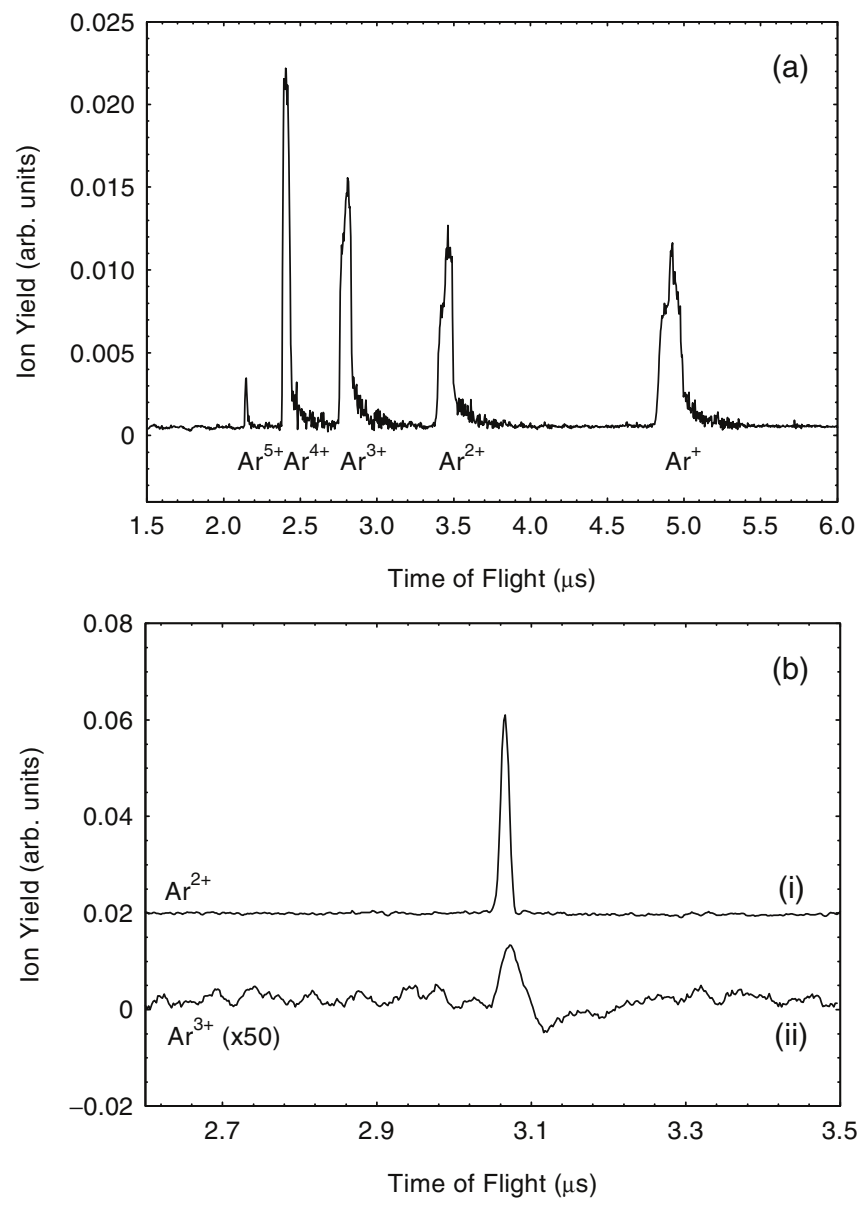

FIG. 1. Time-of-flight spectra under similar laser conditions for ionization of (a) neutral $\mathrm{Ar}$ and (b) $\mathrm{Ar}^{+}$beam with the parallel plate analyzer tuned for maximum transmission of (i) $\mathrm{Ar}^{2+}$ and (ii) $\mathrm{Ar}^{3+}$ (50 times vertical scaling).

from zero [27]. For this reasoning to be valid the ground state peak at $z=0$ must disappear rapidly compared to the peaks at $z= \pm 12$ as the intensity decreases.

That this is indeed the case can be seen from Fig. 2(b), where the peak intensity has been decreased to a value of $5 \times 10^{15} \mathrm{~W} \mathrm{~cm}^{-2}$. While the signal strength of the ionized metastables has decreased by approximately a factor of 3 , the ground state signal has decreased to the extent that it is only just discernible above the metastable signal at the zero lens position. The slight shift in the $z$ position of the ionized metastable peaks from $z=12$ to $z=8$ as the intensity changes from $10^{16}$ to $5 \times 10^{15} \mathrm{~W} \mathrm{~cm}^{-2}$ is an effect of the changes in the intensity-dependent confocal volume. The origin of these peaks is confirmed by the production rates of $\mathrm{Ar}^{3+}$. When the lens is positioned at $z=0$, where the laser focus falls on the ion beam, $\mathrm{Ar}^{3+}$ ions are produced while at $z=12$, where the intensity is lower at the target, no signal is obtained. The signal intensities of the observed $\mathrm{Ar}^{2+}$ peaks at $z=0$ and $z=12$ were monitored as a function of laser intensity. Given that the overlap volume of the mutually orthogonal ion and laser beams varies 


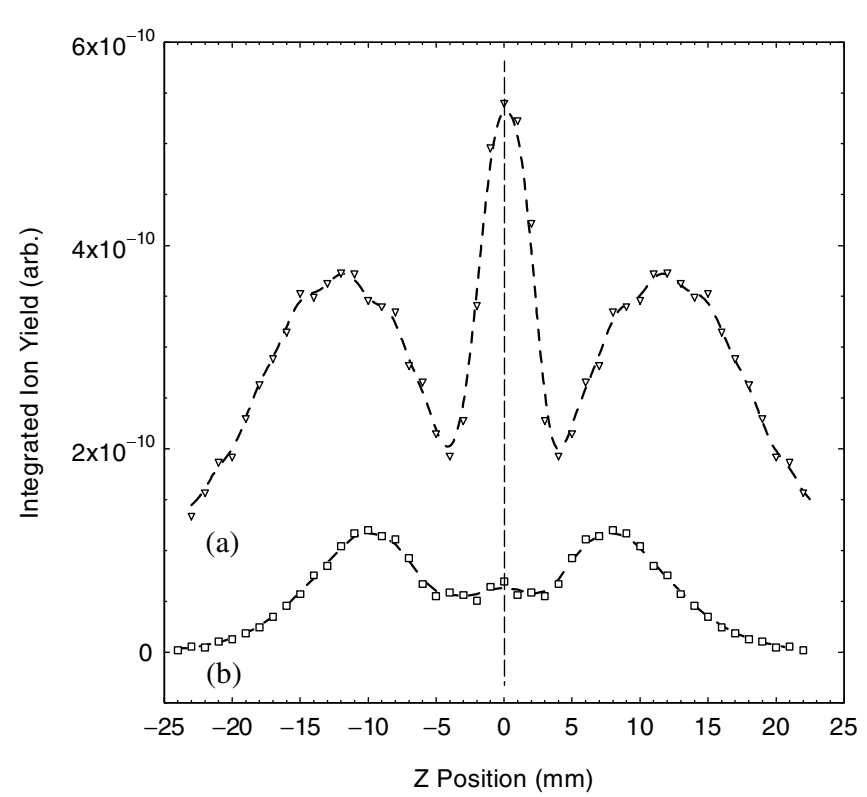

FIG. 2. $\mathrm{Ar}^{2+}$ production from an $\mathrm{Ar}^{+}$ion beam as a function of focusing lens position $(z)$ with respect to the spectrometer detection axis for peak laser intensities: (a) $10^{16} \mathrm{~W} \mathrm{~cm}^{-2}$ and (b) $5 \times 10^{15} \mathrm{~W} \mathrm{~cm}^{-2}$. Mechanical limits of the $z$-scan apparatus restrict the $z$ data collection range from -2 to $23 \mathrm{~mm}$. Data outside this range are shown by the reflection about the focal axis.

with $z$, the signals at $z=0$ and $z=12$ must be normalized. To a good approximation the laser beam may be treated as a cylinder with radius $\omega(z)=1.6 \times 10^{-2} \mathrm{~mm}$ at $z=0$ and $3.7 \times 10^{-1} \mathrm{~mm}$ at $z=12$, where $\omega(z)=$ $\omega_{0} \sqrt{1+\left(z / z_{0}\right)^{2}}$ and $\omega_{0}=2 f \lambda / D$ is the Gaussian beam waist with diameter $D$ prior to focusing. The $1.5 \mathrm{~mm}$ diameter ion beam defines the limits of the interaction volume along the $z$ axis.

Figure 3 shows the measured ion yield with the low intensity curve $\left(10^{11} \rightarrow 10^{13} \mathrm{~W} \mathrm{~cm}^{-2}\right)$ obtained at a focal detuning of $z=12$, whereas the higher intensity curve $\left(10^{14} \rightarrow 10^{16} \mathrm{~W} \mathrm{~cm}^{-2}\right)$ was obtained with the lens positioned so as to focus at $z=0$. It should be noted that the lower intensity $\mathrm{Ar}^{2+}$ curve arises purely from the ensemble of metastable states. Although each state has a unique threshold and saturation intensity, the spread of the ionization potentials over only $2 \mathrm{eV}$ is insufficient to allow experimental resolution of the separate metastable states. The higher intensity $\mathrm{Ar}^{2+}$ curve arises from ionization of both ground state and metastable $\mathrm{Ar}^{+}$; in essence a mixed beam. The small component of the higher intensity $\mathrm{Ar}^{2+}$ curve lying between $10^{14}$ and $10^{15} \mathrm{~W} \mathrm{~cm}^{-2}$ has a slope of 0.5 which is characteristic of the onset of saturated ionization from the $\mathrm{Ar}^{+}$metastables in the diffraction rings surrounding the Airy disk [28].

Using quisiclassical tunneling (ADK) theory [29] to calculate the ionization rate of ground state $\mathrm{Ar}^{+}$in conjunction with the computed interaction volume, a direct fit to the higher intensity $\mathrm{Ar}^{2+}$ intensity curve has been obtained

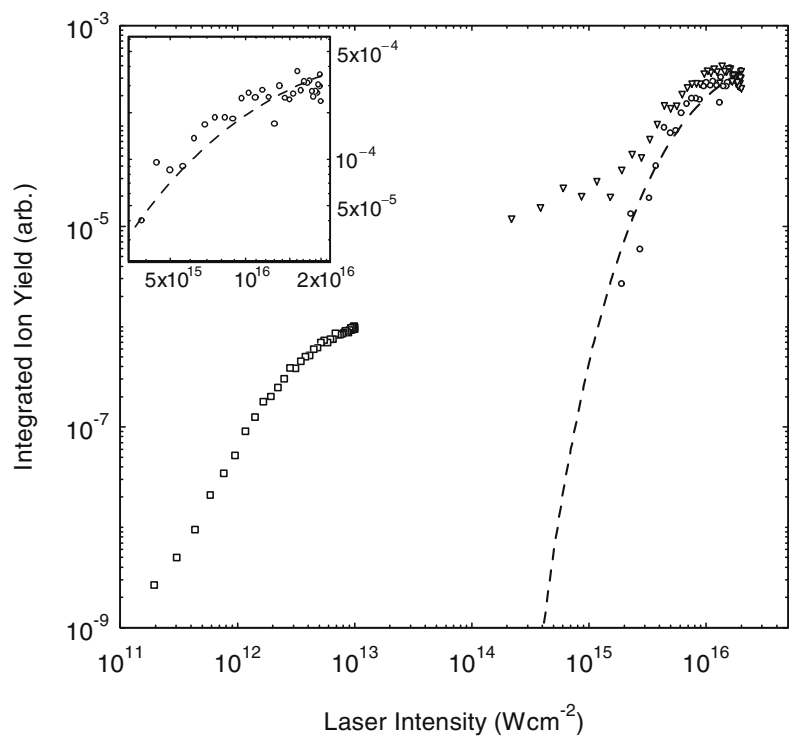

FIG. 3. $\mathrm{Ar}^{2+}$ from an $\mathrm{Ar}^{+}$ion beam as a function of local on-axis peak intensity. Low intensity data (squares) were taken at $z=12$, high intensity data (triangles) at $z=0$ (focus). The circles show the metastable subtracted component representing $\mathrm{Ar}^{2+}$ from ground state $\mathrm{Ar}^{+}$, and the dashed curve shows the ADK fit. An expanded view of this fit is shown in the inset.

after subtraction of the estimated metastable contribution (Fig. 3). The value of the effective principle quantum number $n^{*}=1.44$ used to fit the $\mathrm{Ar}^{+}$ground state signal is in good agreement with the accepted value of $n^{*}=1.4$, confirming the interpretation of our intensity plot.

Multiple ionization of rare gas atoms has been studied theoretically by Becker and Faisal [30] using intense-field many-body $S$-matrix theory which takes into account the electron correlations and the nonperturbative field interactions. The recollision electron impact ionization cross sections used in the calculations are determined from the Lotz formula [31] and the empirical formulations of Fisher et al. [32]. These results, integrated over experimental volumes, are in good agreement with a large number of experimental investigations once normalized to the experimental saturation intensity. This is confirmed for Ar when compared to the benchmark work of Larochelle et al. [33]. An exception is $\mathrm{Ne}$, where there is more than an order of magnitude difference in the ratio of single to double ionization. Previous experimental work on triple ionization of Ar, by Larochelle et al. [33] and Auguste et al. [34], suggests that nonsequential processes are strongly affected by wavelength but not by the pulse length. Our work at $790 \mathrm{~nm}$ is at the same wavelength but with a pulse length about 3 times shorter than that used by Larochelle et al. and consequently, for comparison, by Becker and Faisal.

While the different pulse lengths and interaction volumes forbid a fully quantitative comparison, it is still instructive to compare the present data to the results of Becker and Faisal. It is particularly helpful that they have 
calculated rates for multiple ionization of $\mathrm{Ar}^{+}$as well as for the neutral atom.

Close to the upper limit of the nonsequential intensity regime, Becker and Faisal predict an $\mathrm{Ar}^{3+} / \mathrm{Ar}^{2+}$ ratio of 0.04 , from an initial Ar target, and 0.03, from an initial $\mathrm{Ar}^{+}$target. However, at a corresponding intensity an ADK prediction for purely sequential ionization gives a ratio of 0.01 , much closer to the present value of 0.007 . Thus, while the present measurement from a neutral Ar target confirms the dominance of nonsequential processes in $\mathrm{Ar}^{3+}$ production, such effects appear to be absent in the ion beam measurement. These results show that the removal of an electron, prior to interaction with the laser pulse, almost completely suppresses the nonsequential processes, leaving a small contribution from sequential ionization. This suggests that the initial charge state of the target plays a dominant role in determining the mechanisms for multiple ionization, in contradiction to previous predictions.

This work has opened up experimental studies of multiple ionization in a strong laser field, which to date has been limited to a small range of neutral atoms, to the use of positive ions for direct study. In this initial study we have shown that there are dramatic differences between the strong field ionization of neutral and singly charged Ar, demonstrating the need for further experimental and theoretical work for positive ion targets. Experimental investigations into the interaction of superintense laser pulses $\left(10^{16}-10^{18} \mathrm{~W} \mathrm{~cm}^{-2}\right)$ on targets with even higher ionization potentials (i.e., multiply charged ions) will be possible in the near future. This regime opens up the possibilities of studying relativistic effects, the influence of the laser's magnetic field, and harmonic generation of $\mathrm{keV}$ photons through the nonlinear interaction of electron wave packets oscillating about a strong ionic core.

We thank Hugo van der Hart and Andreas Becker for valuable discussions in the interpretation of our data. This work was funded by the Engineering and Physical Sciences Research Council.

[1] See, for example, K. Codling, J.H. Posthumus, and L. J. Frasinski, in The Physics of Electronic and Atomic Collisions, edited by Y. Itikawa, AIP Conf. Proc. No. 500 (AIP, New York, 2000), pp. 107-112, and references therein.
[2] I. D. Williams et al., J. Phys. B 33, 2743 (2000).

[3] K. Sändig, H. Figger, and T. W. Hansch, Phys. Rev. Lett. 85, 4876 (2000).

[4] A. L'Huiller et al., Atoms in Intense Laser Fields, edited by M. Gravrilla (Academic, New York, 1992), p. 139.

[5] M. Protopapas, C. H. Keitel, and P. L. Knight, Rep. Prog. Phys. 60, 389 (1997).

[6] L. F. DiMauro and P. Agostini, Adv. At. Mol. Opt. Phys. 35, 79 (1995).

[7] P. Paul et al., Science 292, 1689 (2001).

[8] M. Hentschel et al., Nature (London) 414, 509 (2001).

[9] M. Casu, C. Szymanowski, H. Suxing, and C. H. Keitel, J. Phys. B 33, L411 (2000).

[10] S.X. Xu and C.H. Keitel, Phys. Rev. A 63, 053402 (2001).

[11] C. J. Joachain, M. Dorr, and N. Kylstra, Adv. At. Mol. Opt. Phys. 42, 225 (2000).

[12] P. Lambropoulos, P. Margagakis, and J. Zhang, Phys. Rep. 305, 203 (1998).

[13] M. Y. Kuchiev, JETP Lett. 45, 404 (1987).

[14] P. B. Corkum, Phys. Rev. Lett. 71, 1994 (1993).

[15] H. van der Hart and K. Burnett, Phys. Rev. A 62, 013407 (2000).

[16] R. Moshammer et al., Phys. Rev. Lett. 84, 447 (2000).

[17] T. Weber et al., Phys. Rev. Lett. 84, 443 (2000).

[18] R. Lafon et al., Phys. Rev. Lett. 86, 2762 (2001).

[19] A. Becker and F. H. M. Faisal, J. Phys. B 29, L197 (1996).

[20] D. Dundas, K. T. Taylor, J. S. Parker, and E. S. Smyth, J. Phys. B 32, L231 (1999).

[21] H. van der Hart, J. Phys. B 33, L699 (2000).

[22] G. L. Yudin and M. Yu. Ivanov, Phys. Rev. A 63, 033404 (2001).

[23] V. R. Bhardwaj et al., Phys. Rev. Lett. 86, 3522 (2001).

[24] H. Maeda, M. Dammasch, U. Eichmann, and W. Sandner, Phys. Rev. A 63, 025401 (2001).

[25] W. A. Bryan et al., J. Phys. B 33, 745 (2000).

[26] H. D. Hagstrum, Phys. Rev. 104, 309 (1956).

[27] P. Hansch, M. A. Walker, and L. D. Van Woerkom, Phys. Rev. A 54, 4 (1996).

[28] L. Zhang, L. J. Frasinski, and K. Codling, J. Phys. B 27, 3427 (1994).

[29] M. V. Ammosov, N. B. Delone, and V. P. Krainov, Zh. Eksp. Teor. Fiz. 91, 2008 (1986).

[30] A. Becker and F. H. M. Faisal, J. Phys. B 32, L335 (1999).

[31] W. Lotz, Z. Phys. 216, 241 (1968).

[32] V. Fisher et al., J. Phys. B 28, 3027 (1995).

[33] S. Larochelle, A. Talebpour, and S. L. Chin, J. Phys. B 31, 2101 (1998).

[34] T. Auguste et al., J. Phys. B 25, 4181 (1992). 